\title{
Element-specific quantitative determination of the local atomic order in CoPt alloy nanoparticles: Experiment and theory
}

\author{
N. Blanc,,${ }^{1,}$ L. E. Díaz-Sánchez, ${ }^{2, \dagger}$ A. Y. Ramos,${ }^{3}$ F. Tournus,,${ }^{1,}$ H. C. N. Tolentino, ${ }^{3}$ M. De Santis,${ }^{3}$ O. Proux, ${ }^{4}$ A. Tamion, ${ }^{1}$ \\ J. Tuaillon-Combes, ${ }^{1}$ L. Bardotti, ${ }^{1}$ O. Boisron, ${ }^{1}$ G. M. Pastor, ${ }^{2}$ and V. Dupuis ${ }^{1}$ \\ ${ }^{1}$ Institut Lumière Matière, UMR5306 Université Lyon 1-CNRS, Université de Lyon 69622 Villeurbanne cedex, France \\ ${ }^{2}$ Institut für Theoretische Physik, Universität Kassel, 34132 Kassel, Germany \\ ${ }^{3}$ Institut Néel, CNRS and Université Joseph Fourier, 38042 Grenoble, France \\ ${ }^{4}$ Observatoire des Sciences de l'Univers, 38051 Grenoble, France \\ (Received 16 January 2012; published 12 April 2013)
}

\begin{abstract}
An annealing-driven transition from a chemically disordered $A 1$-like structure to a chemically ordered $L 1_{0}$-like phase has been revealed for size-selected CoPt clusters with diameters from 2 to $4 \mathrm{~nm}$, from experiment to theory confrontation. For chemically ordered particles, atypical lattice-parameter ratios $c / a$ are inferred. This ratio is found to be remarkably different depending on the approach used $(c / a>1$ from x-ray absorption at the Co $K$ edge and $c / a \simeq 0.94$ from the Pt dominated x-ray diffraction). A first-principles theoretical study accurately explains this astonishing feature and provides a detailed understanding of the element-specific local relaxations, which are crucial in these nanoalloys.
\end{abstract}

DOI: 10.1103/PhysRevB.87.155412

PACS number(s): 61.46.-w, 61.05.cj, 75.50.Cc

\section{INTRODUCTION}

Magnetic alloy nanoparticles (NPs) unfold novel perspectives of tuning the material performances, for example, in order to control the magnetic anisotropy energy (MAE), thereby pushing the ultimate limit of high-density storage. ${ }^{1-6} \mathrm{CoPt}$ and FePt alloys in the chemically ordered $L 1_{0}$ phase are most promising in this context due to the alternate stacking of a ferromagnetic $3 d$ transition metal with the highly polarizable Pt atoms along the (001) direction. The synthesis and characterization of these nanoalloys have therefore undergone a considerable development in the last decade. ${ }^{5-10}$ Indeed, recent progress has provided access to samples of magnetic NPs with controlled shape, stoichiometry, high stability, and very narrow size distributions, thus rendering nanoscopic material design ultimately possible. The physical synthesis routes (e.g., cluster-beam deposition) preserve the nanometric particle size by embedding them in a solid-state matrix. However, these methods usually lead to chemically disordered structures. One may then attempt to achieve the most interesting $L 1_{0}$ ordered phase, from the disordered $A 1$ phase, by means of annealing. Such post-deposition treatments have been actually performed on clusters as small as 2-4 nm. ${ }^{8-13}$ In spite of this, the expected important enhancement of the MAE remains so far absent. ${ }^{5,13-16}$ The reasons behind these disappointing results are still under debate. ${ }^{13,17-20}$ Taking into account that the magnetization and magnetic anisotropy resulting from $3 d-5 d$ proximity effects are very sensitive to the local environment of the atoms, ${ }^{20-24}$ it is clear that a detailed characterization of the underlying structural and chemical order in nanoalloys is a fundamental requisite for progress in this field.

In this article we demonstrate that CoPt NPs (2-4 nm in diameter) which undergo an annealing-driven transition to the $L 1_{0}$ phase, show nontrivial size-dependent structural relaxations. A combination of leading-edge experimental and theoretical techniques provides an element-specific quantitative characterization of the structure. New insights into the complex atomic rearrangements occurring in these clusters are revealed, which can be interpreted from a local perspective and which provide the basis for a microscopic understanding of the electronic and magnetic properties.

\section{EXPERIMENTAL STUDY}

Size-selected chemically disordered CoPt clusters are synthesized with a sharp size distribution and embedded in a carbon matrix under ultrahigh vacuum by using the low-energy cluster-beam deposition method. ${ }^{25}$ Mass selection allows us to obtain samples with three narrow size distributions having mean diameters $D=2.0,3.1$, and $3.8 \mathrm{~nm}$. In the following these samples are labeled $S_{2}, S_{3}$, and $S_{4}$, respectively. The size distributions are systematically monitored by means of transmission electron microscopy. ${ }^{11,25}$ Once synthesized, the samples are annealed in vacuum at $600^{\circ} \mathrm{C}$ for $2 \mathrm{~h} .{ }^{25,26} \mathrm{All}$ samples present a Gaussian size distribution with a dispersion lower than $8 \%$ and a mean diameter that remains almost unchanged upon annealing. ${ }^{25,27,28}$

Previous high-resolution transmission electron microscopy (HRTEM) images on individual optimally oriented NPs, and the subsequent simulation of the observed contrast, indicate that annealed particles are chemically ordered. ${ }^{11,13}$ In order to get more insight on the annealing-induced changes in the crystalline structure of all the nanoclusters in the sample, we have performed both x-ray diffraction (XRD) and x-ray absorption spectroscopy (XAS) measurements. The XRD data were collected at the CRG-D2AM beam line of the ESRF with a $13 \mathrm{keV}$ photon energy. After annealing, the (111) peak shifts toward lower $q$ values, which implies an increase $(\simeq 1 \%)$ of the distance $d_{111}$, in agreement with HRTEM measurements, where a $1.2 \%$ increase has been determined. ${ }^{29}$ This behavior is opposite to what happens upon chemical ordering for the bulk. ${ }^{30}$ For the $\mathrm{S}_{3}$ sample, by fitting the XRD spectrum ${ }^{29}$ we also find a strong tetragonalization of the cell with a lattice parameter ratio $c / a(\mathrm{XRD})=d_{002} / d_{200}=0.94$. This contrasts with the bulk value $c / a=0.97^{31}$ and demonstrates the impact of size reduction on the crystalline structure.

XAS at the Co $K$ edge $(7709 \mathrm{eV})$ has been performed at room temperature on the CRG BM30b-FAME beam line of the 


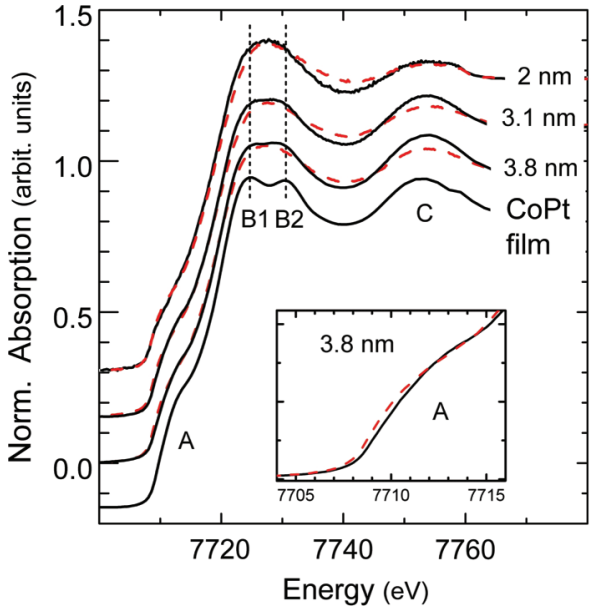

FIG. 1. (Color online) XANES spectra for the $S_{2}, S_{3}$, and $S_{4}$ samples before annealing (red dashed lines) and after annealing (solid lines). The XANES spectrum of a CoPt film is shown for the sake of comparison. The vertical dashed lines indicate the position of the $B 1$ and $B 2$ structures. The inset shows an enlargement of the $A$ shoulder for the $4 \mathrm{~nm}$ sample, before and after annealing.

ESRF. $^{32,33}$ In Fig. 1 the $x$-ray absorption near-edge structure (XANES) both before and after annealing are compared with the reference spectrum of a thick $\operatorname{CoPt} L 1_{0}$ film. ${ }^{34}$ The shoulder $A$ is assigned to transitions from the $1 s$ levels to the unoccupied states mainly originating from the $4 p$ orbitals. In bulk systems this feature varies upon alloying $\mathrm{Co}$ and $\mathrm{Pt}$, reflecting a population rearrangement between the electronic levels. ${ }^{34} \mathrm{~A}$ drop of the $A$ shoulder is expected to be concomitant of a lowering of the $4 p$ band at Co sites with increasing number of Pt neighbors. Such a drop is clearly observed for the annealed samples (see the inset of Fig. 1). The band structure effect for bulk alloy compounds is observed as a split of the white line B, while the nonannealed samples show a broad white line. ${ }^{3,34}$ In nonoriented samples the splitting and the relative amplitudes of these peaks are almost independent of the chemical order in the sample $\left(A 1\right.$ or $\left.L 1_{0}\right)$. In $S_{3}$ and $S_{4}$ we observe a broadening of the top of the $B$ line upon annealing, which is an early sign of the splitting associated to band formation (see $B 1$ and $B 2$ on Fig. 1). This is likely to be related to an increase in the local atomic order around an average Co central atom. This interpretation is confirmed in the larger clusters (3.1 and $3.8 \mathrm{~nm}$ ) by a significant increase in the amplitude of the first EXAFS oscillation $C$ (see Fig. 1).

The structural changes of the clusters upon annealing are also clearly seen in the extended range shown in Fig. 2. The first peak in the Fourier transform (FT) corresponds to the contribution of the first nearest-neighbor (NN) shell. After annealing this peak is shifted toward higher distances, showing an increase in the average $\mathrm{NN}$ distance in the clusters. The increase of intensity in this peak is related to the decrease of structural disorder. The associated modification of the shape of the real part of the FT also indicates a change in the local chemical environment. This evolution must be due to the $A 1 \rightarrow L 1_{0}$ chemical ordering.

A quantitative EXAFS analysis is performed by fitting the $k^{2}$-weighted function $\chi(k)$ to the standard EXAFS formula, using the Artemis software ${ }^{35}$ and focusing on the

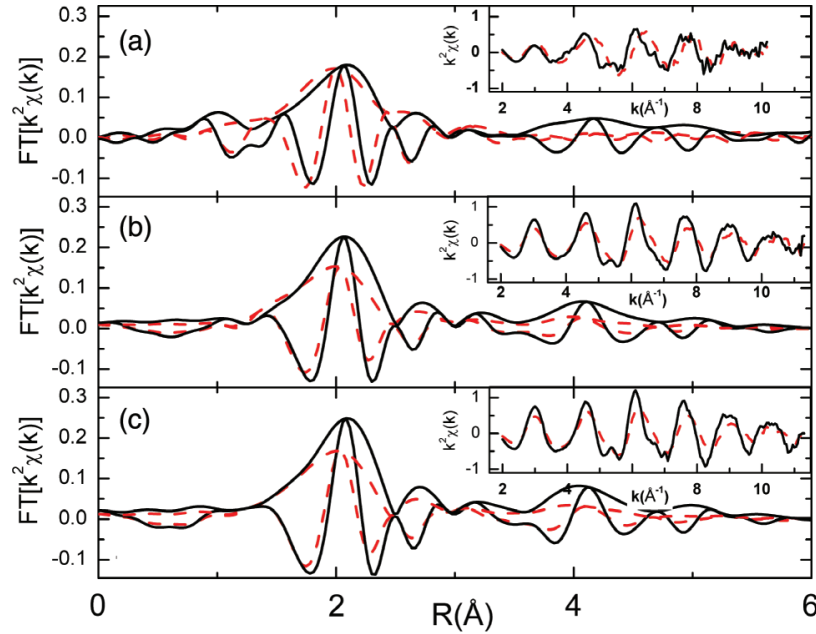

FIG. 2. (Color online) Modulus and real part of the Fourier transform of the EXAFS signal in the various samples: (a) $S_{2}$, (b) $\mathrm{S}_{3}$, and (c) $\mathrm{S}_{4}$. Dashed (solid) curves refers to measurements before (after) annealing. The insets show the corresponding $k^{2}$-weighted EXAFS signals.

NN contribution. Debye-Waller (DW) parameters are used to account for bond-length dispersion, the empirical $S_{0}^{2}$ parameter which reflects many-body losses is kept fixed to an optimized value of $0.75 .^{36}$ The edge energy $E_{0}$ is allowed to slightly vary for the different samples but is taken to be the same for the Pt and Co neighboring shells. A Co atom is indeed surrounded by $N_{\mathrm{Co}}$ cobalt atoms at a distance $d_{\mathrm{CoCo}}$ and $N_{\mathrm{Pt}}$ platinum atoms at a distance $d_{\mathrm{CoPt}}$. As expected for the chemically disordered phase, we find that a ratio $N_{\mathrm{Pt}} / N_{\mathrm{Co}}=1$ can successfully fit the experimental signal for as-prepared particles, while $N_{\mathrm{Pt}} / N_{\mathrm{Co}}=2$ is needed for annealed samples. This shows that the NPs evolve from a complete chemical disorder to a high degree of $L 1_{0}$ order. Then, by keeping these ratios fixed, we can determine and compare the $\mathrm{NN}$ distances $d_{\mathrm{CoCo}}$ and $d_{\mathrm{CoPt}}$ and DW parameters for the different samples. Note that for the $\mathrm{S} 2$ sample a clear conclusion is more difficult to reach since the signal is less intense, there are more than 50\% surface atoms, ${ }^{37}$ and the particles may be less ordered. $8,12,38,39$

For all samples, a drop in the DW factor upon annealing is found, which is consistent with the already discussed global structural ordering within the clusters. Interestingly, the DW parameter $\sigma^{2}$ remains unusually large $\left(\simeq 10^{-2} \AA^{2}\right)$ for both Co-Co and Co-Pt bonds. This is the signature of a significant dispersion of the NN distances. ${ }^{40}$ From best fits [see Fig. 3(a)] we find that in all cases $d_{\mathrm{CoCo}}$ and $d_{\mathrm{CoPt}}$ increase upon annealing (see Table I). Moreover, $d_{\mathrm{CoPt}}$ is always larger than $d_{\mathrm{CoCo}}$. An apparent $c / a$ tetragonalization ratio can be inferred from theses distances, assuming a face-centered tetragonal (fct) crystal structure for the annealed particles: $c / a(\mathrm{XAS})=\sqrt{2\left(d_{\mathrm{CoPt}} / d_{\mathrm{CoCo}}\right)^{2}-1}$. Surprisingly, the calculated $c / a(\mathrm{XAS})$ are significantly larger than 1 . This contrasts not only with the bulk behavior, but also with the outcome of XRD measurements on the same annealed clusters, which are dominated by the scattering contribution from Pt atoms. As shown in Fig. 3(b), a $0.94 c / a$ ratio such as the one deduced from XRD is by no means able to reproduce the experimental curve. 

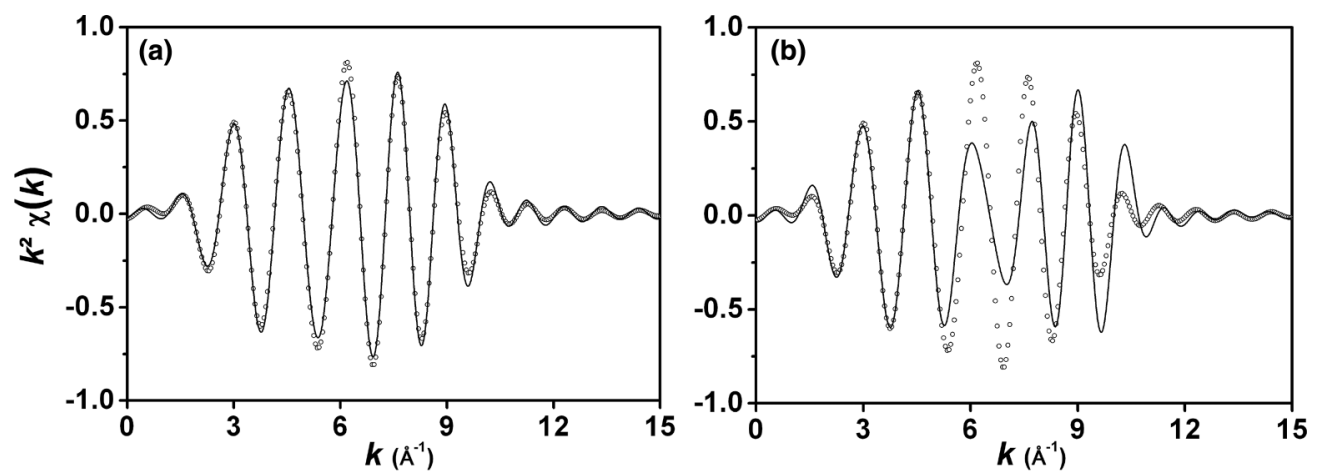

FIG. 3. Comparison between the experimental EXAFS signal (dots, contribution of the NN peak only) and theoretical fits (solid lines) with $c / a(\mathrm{XAS})=1.03(\mathrm{a})$ and $c / a(\mathrm{XAS})=0.94(\mathrm{~b})$ for the annealed $\mathrm{S}_{3}$ sample.

The previous conclusions are not affected by changes in the analysis conditions, even if the edge energy $E_{0}$ is allowed to vary independently for Pt and Co shells, as it can be found in the literature. ${ }^{3,41}$ In fact the experimental trends are so general and robust that they cast doubts on the validity of the assumed perfect fct crystalline order in CoPt clusters. Moreover, the fact that for as-prepared particles $d_{\mathrm{CoCo}}$ is smaller than $d_{\mathrm{CoPt}}$ (as already reported in Refs. 41 and 42) also implies that they are not perfect fcc crystals. This shows that significant relaxation must occur as the result of size reduction. It would then be more accurate to refer to the structures of these NPs as $A 1$-like and $L 1_{0}$-like.

\section{THEORETICAL CALCULATIONS}

In order to clarify the situation from a microscopic perspective we have performed a first-principles theoretical study of the structure of $L 1_{0} \mathrm{CoPt} N P$ s. The calculations have been performed by using the Vienna $a b$ initio simulation package, ${ }^{29,43-45}$ which implements Hohenberg-Kohn-Sham's density-functional theory (DFT) on a periodic supercell and allows fully self-consistent unconstrained structural relaxations. The effects of electron-core interactions are approximated by means of Böchel's projector augmented wave method, ${ }^{46}$ which effectiveness for transition-metal compounds has been largely demonstrated. Exchange and correlation effects are treated by using Perdew and Wang's generalized-gradient approximation. ${ }^{47}$ The ground-state total $S_{z}$, the associated magnetic order, and the cluster geometry are determined on the same footing. ${ }^{29,48}$ This is important since the interatomic distances and magnetic configuration are known to depend strongly on each other.

TABLE I. Interatomic distances (in $\AA$, with a $0.01 \AA$ error bar) and lattice-parameter ratios $c / a$ deduced from EXAFS fits for the different NP samples.

\begin{tabular}{lcccccc}
\hline \hline & \multicolumn{2}{c}{ As-prepared } & & \multicolumn{3}{c}{ Annealed } \\
\cline { 2 - 3 } \cline { 5 - 7 } & $d_{\text {CoCo }}$ & $d_{\text {CoPt }}$ & & $d_{\text {CoCo }}$ & $d_{\text {CoPt }}$ & $c / a(X A S)$ \\
\hline $\mathrm{S}_{2}$ & 2.50 & 2.57 & & 2.52 & 2.64 & 1.09 \\
$\mathrm{~S}_{3}$ & 2.52 & 2.58 & & 2.57 & 2.62 & 1.03 \\
$\mathrm{~S}_{4}$ & 2.53 & 2.59 & & 2.57 & 2.64 & 1.05 \\
\hline \hline
\end{tabular}

Before discussing the cluster results it is worth mentioning that we have determined the equilibrium structure of the bulk $L 1_{0}$ phase of CoPt and found $a=3.82 \AA$ and $c=3.73 \AA$, which corresponds to $d_{\mathrm{CoCo}}=a / \sqrt{2} \simeq 2.70 \AA$ and $c / a=$ 0.97 , in very good agreement with experiment. ${ }^{31}$ The method and parameter choice should therefore provide an accurate description of the structural trends in CoPt nanoalloys. ${ }^{29}$ The relaxed geometry of chemically disordered NPs (truncated octahedral structures with $N=38,201$, and 586 atoms, which cover the size range of samples $S_{2}$ and $S_{3}$ ) has been determined. The interatomic distances are in excellent agreement with the EXAFS results. We have then studied the same CoPt clusters with a perfect $L 1_{0}$ chemical order. In each case a number of different initial lattice parameters have been considered as starting points of the unconstrained optimization with respect to $S_{z}$ and cluster geometry. The relaxed average interatomic distances and $c / a$ ratios at the $\mathrm{Co}$ and $\mathrm{Pt}$ sites (calculated from $d_{\mathrm{CoPt}}, d_{\mathrm{CoCo}}$, and $d_{\mathrm{PtPt}}$ ) are not affected by the details of the starting geometry. As shown in Table II, our results reproduce remarkably well the outcome of the EXAFS and XRD measurements. They reveal the presence of important environment-dependent relaxations at the Co and $\mathrm{Pt}$ atoms, thereby removing the apparent inconsistency between the XRD and EXAFS experiments. Notice in particular the contrasting values $c / a>1$ and $c / a \leqslant 0.95$ at the Co and $\mathrm{Pt}$ sites, and their convergence to the bulk limit as $N$ increases.

In order to gain further insight into the structural order in $L 1_{0}$-like clusters it is useful to analyze the relaxed structures from a local perspective. In Fig. 4 we consider $\mathrm{Co}_{300} \mathrm{Pt}_{286}$ as a representative example $(D \simeq 2.6 \mathrm{~nm})$. While the relaxed

TABLE II. Average interatomic distances (in $\AA$ ) and latticeparameter ratios $c / a(\mathrm{Co})$ [to be compared to $c / a(\mathrm{XAS})$ ] and $c / a(\mathrm{Pt})$ [to be compared to $c / a(\mathrm{XRD})$ ] of relaxed $L 1_{0}$ CoPt clusters having $N$ atoms, as obtained from DFT calculations. For $N=586$, the corresponding standard deviation $\sigma$ of $d$ is given in parentheses.

\begin{tabular}{lccccc}
\hline \hline$N$ & $d_{\text {CoCo }}$ & $d_{\mathrm{CoPt}}$ & $d_{\mathrm{PtPt}}$ & $c / a(\mathrm{Co})$ & $c / a(\mathrm{Pt})$ \\
\hline 38 & 2.43 & 2.60 & 2.75 & 1.13 & 0.89 \\
201 & 2.60 & 2.63 & 2.71 & 1.02 & 0.94 \\
586 & 2.64 & 2.64 & 2.70 & 1.00 & 0.95 \\
& $(0.17)$ & $(0.05)$ & $(0.05)$ & & \\
Bulk & 2.70 & 2.66 & 2.70 & 0.97 & 0.97 \\
\hline \hline
\end{tabular}


(b)
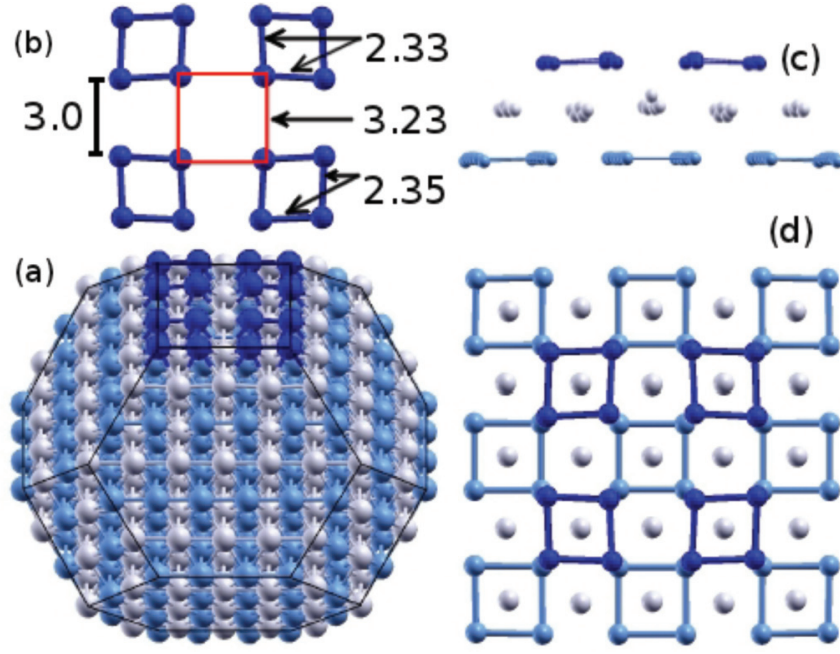

FIG. 4. (Color online) Illustration of the relaxed $L 1_{0}$-like $\mathrm{Co}_{300} \mathrm{Pt}_{286}$ cluster (a), where dark and light spheres represent $\mathrm{Co}$ and Pt atoms, respectively. (b) Top view along the (001) direction of the uppermost Co facet [highlighted in (a)]. The interatomic distances are given in $\AA$. (c) Side view of the three uppermost Co and $\mathrm{Pt}$ planes showing the important Pt-layer corrugation. (d) Top view of the three uppermost $\mathrm{Co}$ and $\mathrm{Pt}$ layers along the (001) direction. Notice the tendency of the Co atoms to form short-bond-length squares (tetramerization).

cluster preserves the overall truncated-octahedral shape and $L 1_{0}$ chemical order [an indication of its stability, see Fig. 4(a)] a more detailed analysis shows that the equilibrium positions follow quite different patterns in the alternating (001) layers. Quite generally, and in contrast to the bulk structure, the Co and Pt layers appear to relax independently from each other, despite the strong CoPt hybridization. In fact, these different relaxations of $\mathrm{Co}$ and $\mathrm{Pt}$ atoms are responsible for the different $c / a$ ratios observed in experiments. For instance, in the uppermost (001) Co layer the Co atoms show a clear in-plane tetramerization, which does not match the underlying Pt layer (see Fig. 4). Moreover, these unusual rearrangements explain the experimentally observed strong dispersion in $d_{\mathrm{CoCo}}$ $\left(2.33 \leqslant d_{\mathrm{CoCo}} \leqslant 3.23 \AA\right.$ for this layer). The tendency of Co NN bonds to be shorter than the Pt ones results in structural stress, which in a finite cluster can be more easily reduced by moving the Co atoms within the facets. In the case of Pt, however, there is less room for in-plane relaxation and therefore the changes in the in-plane PtPt distances are smaller. The Pt atoms thus tend to move out of the (001) plane creating important Pt-layer corrugations [see Fig. 4(c)]. Comparing the local densities of states (DOS) before and after relaxation allows us to identify the main electronic sources of the observed relaxations. First, one observes a shift to lower energies of the occupied majority
Co states. Second, the symmetry lowering causes an important depletion of the minority spin Co DOS at $\varepsilon_{F}$, which contrasts with the high DOS in the unrelaxed structure. Tetramerization appears to be a compromise between enhancing CoCo bonds and keeping strong hybridizations with the $\mathrm{NN} \mathrm{Pt}$ atoms of the adjacent layers. Different rearrangements are found at different layers: From a strong tetramerization at the outermost layers to a nearly perfect $L 1_{0}$ order at the cluster center. ${ }^{29}$ Calculations for Pt terminated clusters $(N=201$ atoms $)$ yield similar distributions of interatomic distances and element specific $c / a$ ratios: $d_{\mathrm{CoCo}}=2.63 \AA, d_{\mathrm{CoPt}}=2.62 \AA$, and $d_{\mathrm{PtPt}}=2.69 \AA$, which imply $c / a=1.0$ at the Co atoms and $c / a=0.95$ at the $\mathrm{Pt}$ atoms. See also Table II. Therefore, our conclusions should hold for clusters having both Co and Pt terminated facets. It should also be noted that there is no experimental indication for a strong Pt segregation at the surface or for more probable Pt-terminated facets.

\section{CONCLUSION}

In conclusion, we have shown that CoPt clusters obtained by size-selected cluster-beam deposition with diameter around $3 \mathrm{~nm}$, transform upon annealing into a chemically ordered $L 1_{0}$-like phase. A quantitatively consistent experimental and theoretical determination of the interatomic distances has been achieved. Thus, a remarkable size and element dependence of the local atomic relaxations in the $L 1_{0}$ clusters has been revealed. Special care in the way the lattice parameters are inferred is crucial for a consistent interpretation of different element-specific experiments. A theoretical analysis from a local perspective has provided new insights into the intricate relaxations of the $\mathrm{Co}$ and $\mathrm{Pt}$ atoms which suggests that the observed anomalous $c / a$ ratios are an intrinsic property of CoPt nanoparticles. This finite-size phenomenon is expected to be of major importance for the relative stability of the different cluster structures. ${ }^{12,39,49}$ Moreover, they are likely to affect the $\mathrm{MAE}^{4,23,24,50}$ and may contribute to the previously reported anisotropy lowering, as compared to bulk $L 1_{0}$ CoPt. Similar structural complexities are expected for other $3 d-5 d$ and $3 d-4 d$ nanoalloys and should play a central role in understanding and controlling the ensemble of electronic and magnetic properties of magnetic nanoalloys.

\section{ACKNOWLEDGMENTS}

This work has been supported by GDR-CNRS 3182, DFG, DAAD, and COST Action MP0903. The computer resources were provided by the John von Neumann Institute for Computing. We acknowledge the PLYRA for the cluster synthesis and the French CRG BM02 and BM30B beam lines at the ESRF for providing synchrotron radiation and assistance.

\footnotetext{
*Currently at Institut Néel, CNRS and Université Joseph Fourier, 38042 Grenoble, France.

${ }^{\dagger}$ Currently at Facultad de Ciencias, Universidad Autónoma del Estado de México, Toluca, México.

$\ddagger$ florent.tournus@univ-lyon1.fr

${ }^{1}$ D. J. Sellmyer, M. Yu, and R. D. Kirby, Nanostruct. Mater. 12, 1021 (1999).
}

${ }^{2}$ D. Weller, A. Moser, L. Folks, M. E. Best, W. Lee, M. F. Toney, M. Schwickert, J.-U. Thiele, and M. F. Doerner, IEEE Trans. Magn. 36, 10 (2000).

${ }^{3}$ J. Park, M. Kim, Y. Jun, J. Lee, W. Lee, and J. Cheon, J. Am. Chem. Soc. 126, 9072 (2004).

${ }^{4}$ G. Barcaro, L. Sementa, F. R. Negreiros, R. Ferrando, and A. Fortunelli, Nano Lett. 11, 5542 (2011). 
${ }^{5}$ S. Sun, C. B. Murray, D. Weller, L. Folks, and A. Moser, Science 287, 1989 (2000).

${ }^{6}$ C.-b. Rong, D. Li, V. Nandwana, N. Poudyal, Y. Ding, Z. Wang, H. Zeng, and J. Liu, Adv. Mater. 18, 2984 (2006).

${ }^{7}$ A.-H. Lu, E. L. Salabas, and F. Schüth, Angew. Chem. Int. Ed. 46, 1222 (2007).

${ }^{8}$ D. Alloyeau, C. Ricolleau, C. Mottet, T. Oikawa, C. Langlois, Y. Le Bouar, N. Braidy, and A. Loiseau, Nat. Mater. 8, 940 (2009).

${ }^{9}$ D. Alloyeau, G. Prévot, Y. Le Bouar, T. Oikawa, C. Langlois, A. Loiseau, and C. Ricolleau, Phys. Rev. Lett. 105, 255901 (2010).

${ }^{10}$ F. Tournus, K. Sato, T. Epicier, T. J. Konno, and V. Dupuis, Phys. Rev. Lett. 110, 055501 (2013).

${ }^{11}$ N. Blanc, F. Tournus, V. Dupuis, and T. Epicier, Phys. Rev. B 83, 092403 (2011).

${ }^{12}$ P. Andreazza, C. Mottet, C. Andreazza-Vignolle, J. Penuelas, H. C. N. Tolentino, M. De Santis, R. Felici, and N. Bouet, Phys. Rev. B 82, 155453 (2010).

${ }^{13}$ F. Tournus, A. Tamion, N. Blanc, A. Hannour, L. Bardotti, B. Prevel, P. Ohresser, E. Bonet, T. Epicier, and V. Dupuis, Phys. Rev. B 77, 144411 (2008).

${ }^{14}$ F. Tournus, N. Blanc, A. Tamion, M. Hillenkamp, and V. Dupuis, Phys. Rev. B 81, 220405(R) (2010).

${ }^{15}$ C.-b. Rong, N. Poudyal, G. S. Chaubey, V. Nandwana, R. Skomski, Y. Q. Wu, M. J. Kramer, and J. P. Liu, J. Appl. Phys. 102, 043913 (2007).

${ }^{16}$ X. Sun, Z. Y. Jia, Y. H. Huang, J. W. Harrell, D. E. Nikles, K. Sun, and L. M. Wang, J. Appl. Phys. 95, 6747 (2004).

${ }^{17}$ C. Antoniak, M. Spasova, A. Trunova, K. Fauth, F. Wilhelm, A. Rogalev, J. Minàr, H. Ebert, M. Farle, and H. Wende, J. Phys.: Condens. Matter 21, 336002 (2009).

${ }^{18}$ T. Burkert, O. Eriksson, S. I. Simak, A. V. Ruban, B. Sanyal, L. Nordström, and J. M. Wills, Phys. Rev. B 71, 134411 (2005).

${ }^{19}$ S. Okamoto, N. Kikuchi, O. Kitakami, T. Miyazaki, Y. Shimada, and K. Fukamichi, Phys. Rev. B 66, 024413 (2002).

${ }^{20}$ F. Tournus, S. Rohart, and V. Dupuis, IEEE Trans. Magn. 44, 3201 (2008); S. Rohart, F. Tournus, and V. Dupuis, arXiv:1105.6292.

${ }^{21}$ J. Dorantes-Dávila, H. Dreyssé, and G. M. Pastor, Phys. Rev. Lett. 91, 197206 (2003).

${ }^{22}$ M. Muñoz-Navia, J. Dorantes-Dávila, D. Zitoun, C. Amiens, N. Jaouen, A. Rogalev, M. Respaud, and G. M. Pastor, Appl. Phys. Lett. 95, 233107 (2009).

${ }^{23}$ J. B. Staunton, S. Ostanin, S. S. A. Razee, B. Gyorffy, L. Szunyogh, B. Ginatempo, and E. Bruno, J. Phys.: Condens. Matter 16, S5623 (2004).

${ }^{24}$ R. Cuadrado and R. W. Chantrell, Phys. Rev. B 86, 224415 (2012).

${ }^{25}$ F. Tournus, N. Blanc, A. Tamion, M. Hillenkamp, and V. Dupuis, J. Magn. Magn. Mat. 323, 1868 (2011).

${ }^{26} \mathrm{We}$ have verified that the system does not evolve with longer annealing periods $(6 \mathrm{~h})$. It would be also interesting to explore the possibility of tuning the details of the $L 1_{0}$ structure by varying the annealing parameters, such as the temperature, duration, and quench rate.

${ }^{27}$ F. Tournus, N. Blanc, A. Tamion, V. Dupuis, and T. Epicier, J. Appl. Phys. 109, 07B722 (2011).

${ }^{28}$ In one hand, an amorphous carbon matrix has been chosen because it is an excellent and very stable protection for nanoparticles prepared under UHV by physical way. Moreover, the preferential graphitization of the carbon matrix occurring during thermal annealing offers an even better protection of $L 1_{0} \mathrm{CoPt}$ nanoparticles. This has already been reported for $\mathrm{Co}, \mathrm{Fe}$, and $\mathrm{FePt}$ nanoparticles encapsulated in graphitelike carbon [N. Jaouen, D. Babonneau, J. M. Tonnerre, D. Carbone, F. Wilhelm, A. Rogalev, T. K. Johal, and G. van der Laan, Phys. Rev. B 76, 104421 (2007), and references therein]. On the other hand, the magnetic size of bimetallic CoPt clusters embedded in a carbon matrix is initially the same as the TEM size and remains unchanged upon annealing. In addition, a significant increase of the $\mathrm{Co}$ and $\mathrm{Pt}$ magnetic moments with respect to the bulk moments has been derived from XMCD measurements in both chemically disordered and ordered CoPt clusters. ${ }^{13}$ Consequently, carbon contamination seems highly improbable.

${ }^{29}$ See Supplemental Material at http://link.aps.org/supplemental/ 10.1103/PhysRevB.87.155412 for XRD results, HRTEM $d_{111}$ measurements, and details about the DFT calculations.

${ }^{30}$ C. Leroux, M. C. Cadeville, V. Pierron-Bohnes, G. Inden, and F. Hinz, J. Phys. F: Met. Phys. 18, 2033 (1988).

${ }^{31}$ JCPDS, CoPt, 34-1358.

${ }^{32}$ The storage ring was operated at $6 \mathrm{GeV}$ with $200 \mathrm{~mA}$ current. The energy was selected by using a $\mathrm{Si}(220)$ double-crystal monochromator with dynamic sagittal focusing, which yields a beam spot on the sample having $300 \mu \mathrm{m}$ horizontal by $200 \mu \mathrm{m}$ vertical size and an x-ray flux of $10^{12}$ photons/s.

${ }^{33}$ O. Proux, V. Nassif, A. Prat, O. Ulrich, E. Lahera, X. Biquard, J. Menthonnex, and J. Hazemann, J. Synchrotron Radiat. 13, 59 (2006).

${ }^{34}$ N. M. Souza-Neto, A. Y. Ramos, H. C. N. Tolentino, and Y. Joly, J. Phys.: Conf. Ser. 190, 012112 (2009).

${ }^{35}$ B. Ravel and M. Newville, J. Synchrotron Radiat. 12, 537 (2005).

${ }^{36}$ B. K. Teo, EXAFS: Basic Principles and Data Analysis (Springer, Berlin, 1986).

${ }^{37}$ A. M. Beale and B. M. Weckhuysen, Phys. Chem. Chem. Phys. 12, 5562 (2010).

${ }^{38}$ L. Qin, Y. Zhang, S. Huang, H. Tian, and P. Wang, Phys. Rev. B 82, 075413 (2010).

${ }^{39}$ G. Rossi, R. Ferrando, and C. Mottet, Faraday Discuss. 138, 193 (2008).

${ }^{40}$ G. Greco, A. Witkowska, E. Principi, M. Minicucci, and A. Di Cicco, Phys. Rev. B 83, 134103 (2011).

${ }^{41}$ B. J. Hwang, S. M. S. Kumar, C.-H. Chen, Monalisa, M.-Y. Cheng, D.-G. Liu, and J.-F. Lee, J. Phys. Chem. C 111, 15267 (2007).

${ }^{42}$ F.-J. Lai, L. S. Sarma, H.-L. Chou, D.-G. Liu, C.-A. Hsieh, J.-F. Lee, and B.-J. Hwang, J. Phys. Chem. C 113, 12674 (2009).

${ }^{43}$ G. Kresse and J. Furthmüller, Comput. Mat. Sci. 6, 15 (1996).

${ }^{44}$ G. Kresse and J. Furthmüller, Phys. Rev. B 54, 11169 (1996).

${ }^{45}$ G. Kresse and D. Joubert, Phys. Rev. B 59, 1758 (1999).

${ }^{46}$ P. E. Blöchl, Phys. Rev. B 50, 17953 (1994).

${ }^{47}$ J. P. Perdew, J. A. Chevary, S. H. Vosko, K. A. Jackson, M. R. Pederson, D. J. Singh, and C. Fiolhais, Phys. Rev. B 46, 6671 (1992).

${ }^{48}$ The average magnetic moment per atom $\bar{\mu}_{N}=2 S_{z} / N$ is determined by varying systematically the total spin polarization $S_{z}$ in the complete physically relevant range (typically from $\bar{\mu}_{N}=1 \mu_{B}$ up to fully saturated spin moments). For each fixed value of $S_{z}$ the cluster structure is fully relaxed without imposing any constraints.

${ }^{49}$ M. E. Gruner, G. Rollmann, P. Entel, and M. Farle, Phys. Rev. Lett. 100, 087203 (2008).

${ }^{50}$ S. S. A. Razee, J. B. Staunton, B. Ginatempo, E. Bruno, and F. J. Pinski, Phys. Rev. B 64, 014411 (2001). 